\title{
Análise dos padrões de viagem de um polo gerador de viagens, aplicada ao campus universitário da UFMT, em Cuiabá
}

\section{Analysis of travel patterns of a trip generator applied at UFMT university campus in Cuiabá}

\author{
Belufi', Felipe Nunes; Baltar², Marina Leite de Barros; Bender ${ }^{3}$, Juliane Érika \\ Cavalcante; Chegury 4 , Juliana Queiroz Borges de Magalhães \\ I FAENG/UFMT, Rua Acorizal, 133, CEP 78048-405, Brasil, fbelufi@gmail.com \\ 2 FAENG/UFMT, mabaltar@gmail.com \\ 3FAENG/UFMT, bender@pet.coppe.ufri.br \\ 4 FAENG/UFMT, julianagbchegury@gmail.com
}

\begin{abstract}
RESUMO
O objetivo deste trabalho é analisar os padrões de viagem de usuários de um polo gerador de viagens, quanto à distância dos deslocamentos, ao modo de transporte escolhida e a natureza da viagem. A análise foi realizada no Campus Universitário da UFMT em Cuiabá com dados obtidos por formulário on-line respondidos por usuários desta instituição para avaliar o método da análise quanto a sua eficiência no fornecimento de informações dos padrões de viagem no que tange aos preceitos da mobilidade urbana sustentável. O método se mostrou satisfatório, porém as pesquisas devem ser aprofundadas.
\end{abstract}

Palavras-chave: mobilidade sustentável, Campus Universitário, Polo gerador de viagem.

\begin{abstract}
The objective of this paper is to analyze travel patterns of users of a Trip Generator, as the distance of the travels, to the mode of transport chosen and the nature of the trip. Apply the analysis in the University Campus of the UFMT in Cuiabá, with data obtained by online form answered by users of this institution and evaluate the method of analysis for its efficiency in providing information on travel patterns with respect to the precepts of sustainable urban mobility. The method proved to be satisfactory, but the research should be deepened.
\end{abstract}

Keywords: Sustainable mobility, University Campus, Polo generators travel.

\section{INTRODUÇÃO}

Um polo gerador de viagem (PGV) é definido como local ou instalação de naturezas distintas que desenvolvem atividades de porte e escala capaz de produzir um contingente

${ }^{1}$ BELUFI, Felipe Nunes; BALTAR, Marina Leite de Barros; BENDER, Juliane Érika Cavalcante; CHEGURY, Juliana Queiroz Borges de Magalhães. Análise dos padrões de viagem de um polo gerador de viagens, aplicada ao campus universitário da UFMT, em Cuiabá. In: II SIMPÓSIO NACIONAL DE GESTÃO URBANA: SINGEURB, 2019, São Paulo. Anais... Porto Alegre: ANTAC, 2019. 
significativo de viagens (PORTUGAL e GOLDNER, 2003), sendo assim, instituições de ensino superior (IES) são considerados polos geradores de viagens (REDEPGV, 2019).

A escolha do modo de transporte se faz por uma tomada de decisão pessoal que considera diversos elementos (OLIVEIRA, 2015; PASCHOAL e ARRUDA, 2018). Em geral, essa escolha está relacionada à distância percorrida nos deslocamentos e ao perfil do usuário. Escolhas de modos de transporte não sustentáveis trazem impactos negativos para a mobilidade urbana, demonstrando a relevância de estudar o modo de transporte adotado pelos usuários de um PGV.

Paschoal e Arruda (2018) apontam a distância como um dos principais determinantes nas escolhas do modo de transporte, esses fatores foram identificados com base na frequência e na intensidade em que foram apontados por estudantes da Universidade de Brasilia.

É importante salientar que analisar como as distâncias das viagens determinam a escolha modal se torna totalmente ineficaz se as características pessoais da pessoa não forem também consideradas (Paschoal e Arruda, 2018). Os autores, ainda concluem que, compreender os elementos envolvidos na tomada de decisão frente a escolha do modo de transporte é fundamental para o planejamento urbano.

Takano (2010) argumenta que os deslocamentos diários não são tão simples de entender e os categoriza como podendo ser viagens pendulares (casa - atividade principal - casa), de cadeia simples, ou ainda viagens encadeadas, em que outras atividades/viagens secundárias estão distribuídas entre a casa e a atividade principal.

O fato de o deslocamento ser simples ou encadeado interfere significativamente nas escolhas quanto ao modo de transporte utilizado.

Faz-se necessário investigar os atores relacionados aos PGVs e todas as características que definem sua forma de deslocamento com o intuito de fornecer informações de como essas pessoas se relacionam com o meio ambiente que estão inseridas, visto que parte do problema de mobilidade urbana é atribuída a um mau planejamento dos PGVs devido à falta de informações sobre os padrões de viagem dos usuários (ALVES et al., 2015).

Neste trabalho, propõem-se classificar os padrões de viagens dos usuários do Campus Universitário de Cuiabá, que tem uma população estimada em 13.645 pessoas, entre servidores e discentes (UFMT, 2018). As análises serão feitas a partir do modo de transporte escolhido em função da distância das viagens e da natureza do deslocamento. Com essas análises, busca-se fornecer informações de suporte para definir medidas visando o incentivo a viagens mais sustentáveis.

Os dados para análise foram obtidos através de um questionário on-line sobre a mobilidade no Campus da UFMT realizada entre fevereiro e março de 2019. Os entrevistados foram convidados a participar da pesquisa pelas redes sociais e por e-mail institucional. Foram obtidas 1.639 respostas, representando $12 \%$ da população estimada.

\section{CLASSIFICAÇÃO DO DESLOCAMENTO}

\subsection{Quanto a distância}

Na pesquisa foi questionada a origem dos deslocamentos que tinham a universidade como destino e também o destino das viagens ao sair do Campus. Os entrevistados indicaram a cidade e bairro em que residem e que trabalham, quando este fosse escolhido como uma origem ou destino de algum de seus deslocamentos.

Para o estudo das distâncias de deslocamentos, a cidade foi dividida em zonas com suporte do software Autocad. Para isso, foram traçados círculos de raios pré-determinados tendo a UFMT como o centro. Dessa forma, a distância máxima dos deslocamentos de cada zona, com destino ou origem ao Campus Universitário da UFMT, equivale ao raio do círculo que limita cada zona. 
Uma vez que o centro geométrico de um bairro está dentro de uma dessas zonas, este bairro pertence a esta zona, e a nenhuma outra mais, e, por consequência, aquela zona representa todos os entrevistados que indicaram tal bairro. Isto permite classificar todos os bairros em zonas que representam a distância máxima percorrida pelos usuários em seus deslocamentos que tem destino ou origem o Campus Universitário da UFMT.

Para origem ou destino no município de Cuiabá, a classificação de bairros em zonas foi feita utilizando como base o Mapa de Abairramento de Cuiabá (Prefeitura de Cuiabá/SMDU/DPD, 2010), como mostra a Figura 1. Quando o deslocamento tinha como origem ou destino outro município, como Várzea Grande, utilizou-se o recurso de sobreposição de imagem do software Google Earth, que permite buscar a localização do bairro. A imagem da Figura 1 foi sobreposta à imagem do satélite do software, e os bairros foram classificados de acordo com as zonas hachuradas.

Figura 1 - Zoneamento dos bairros em distância

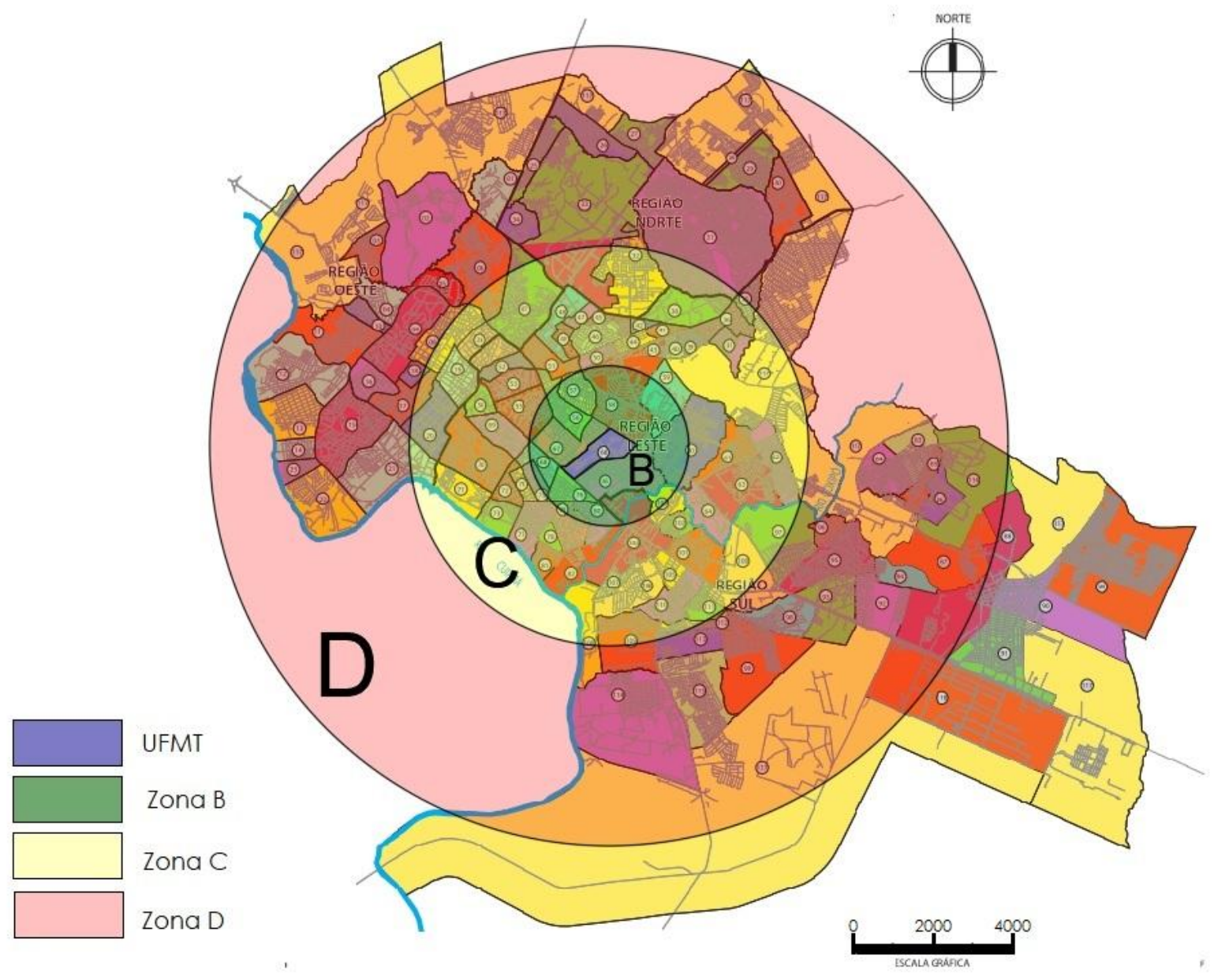

Fonte: Autor (2019)

\subsection{Quanto a sua natureza}

Os entrevistados também foram questionados quanto à natureza do seu deslocamento: casaestudo, trabalho-estudo, casa-trabalho ou outros.

Os deslocamentos para o campus da UFMT que possuem base residencial tanto na origem quanto no destino foram classificados como viagem simples. As viagens foram classificadas como viagem encadeada nos casos em que um dos deslocamentos tem base residencial e o outro deslocamento possui uma origem/destino intermediário. 


\section{RESULTADOS E ANÁLISES}

Após análise dos dados, foi observado que $2 \%$ da amostra tinham resultados incompletos ou errôneos, por falta ou inconsistência da informação de bairro de residência ou de trabalho, ou por se tratar de outros campi da UFMT em cidades diferentes. Desconsiderando esses dados, a amostra passou a ser composta por 1.600 respostas, e não mais por 1.639.

Para análise de resultado foi admitido que 0 maior deslocamento representaria 0 deslocamento padrão das viagens de cada usuário, tanto no modo de transporte e na zona de deslocamento.

De acordo com as respostas obtidas, o transporte público coletivo (TC) é a segunda modalidade de transporte mais utilizada, o modo ônibus é utilizado em 39,19\% das viagens. Sendo que o transporte individual (TI) corresponde a $46,69 \%$ das viagens.

A modalidade de TI compreende os modos: carro, moto, carona e aplicativo de transporte. O maior destaque entre esses modos de transporte é para as viagens de veículos particulares, utilizada por $36,7 \%$ dos usuários do campus, seguida de moto, 6,9\%.

As caronas e aplicativo de transportes são utilizados por $3,1 \%$ dos usuários e, geralmente, estão associados a caminhadas a pé em um dos deslocamentos e/ou viagens encadeadas.

As viagens do modo bicicleta e a pé são da modalidade ativa (TA), que representa $14,13 \%$ das viagens. Desses, a grande maioria são as caminhadas, a bicicleta é pouco utilizada nas escolhas dos usuários do campus.

Na Figura 2 temos a frequência dos modos de transporte escolhidos nas viagens dos usuários, e na Figura 3 a frequência das modalidades de transporte.

Figura 2 - Modo de transporte, em frequência relativa ao total de viagens

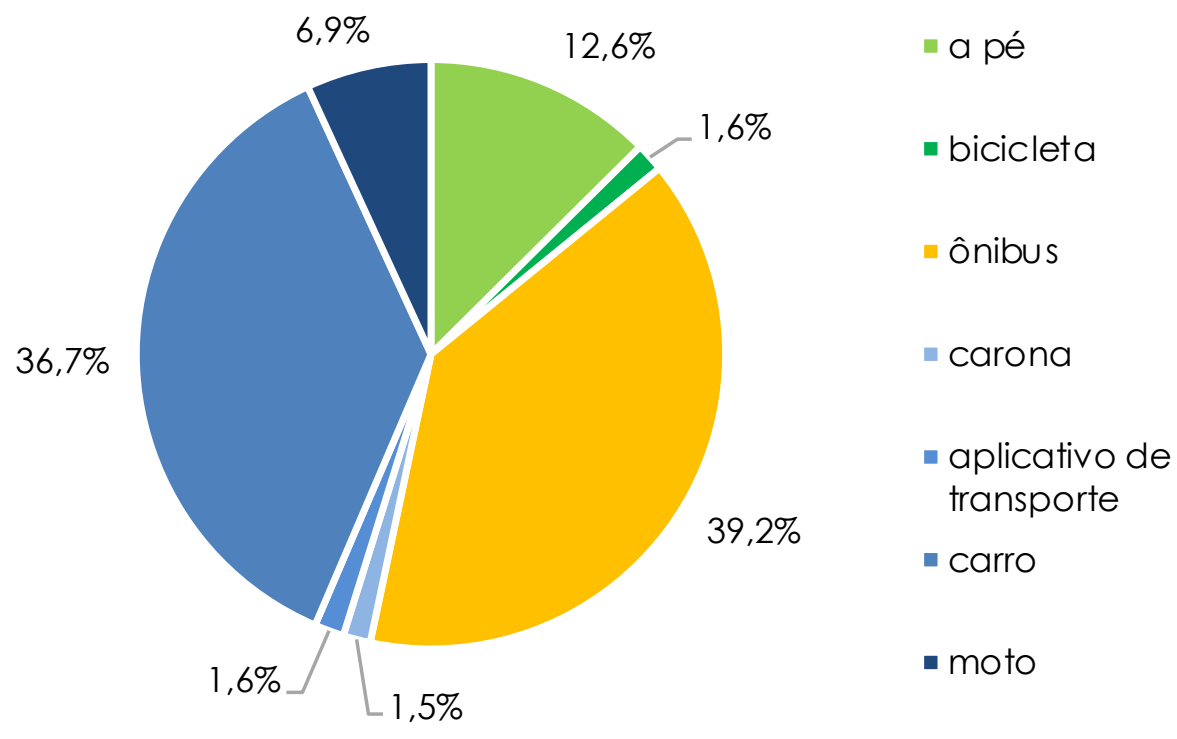

Fonte: Autor (2019) 
Figura 3 - Modalidade de transporte, em frequência relativa ao total de viagens

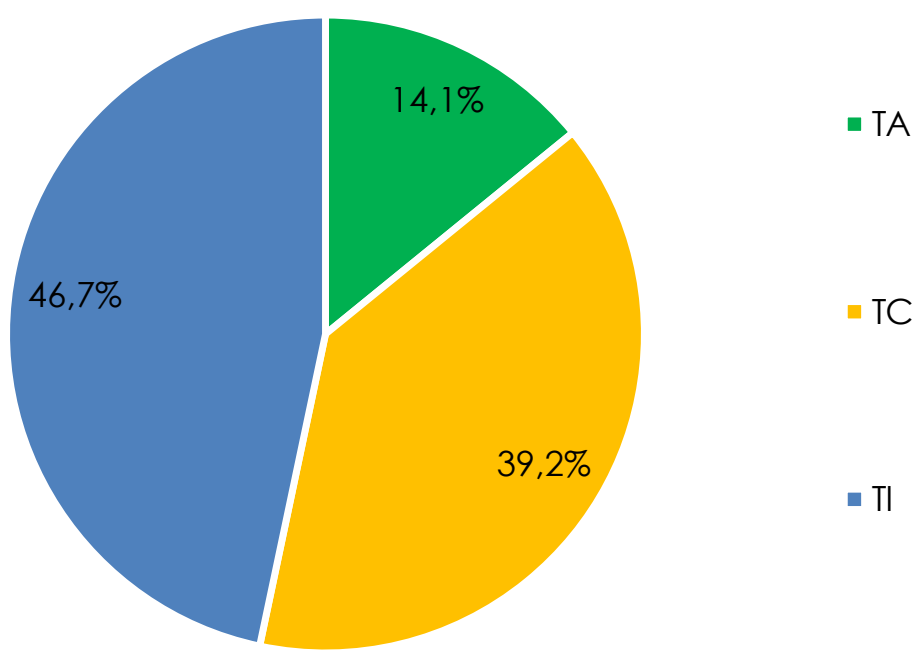

Fonte: Autor (2019)

Quando analisamos as zonas de deslocamento dos usuários, a maior parte $(40,4 \%)$ faz suas viagens da zona $D$, isto é, se deslocam entre 5 e $10 \mathrm{~km}$ para chegar ao Campus. A zona $C$ é responsável por 30,9\% das viagens, com deslocamentos de no máximo $5 \mathrm{~km}$. E 21,2\% dos usuários têm suas viagens num raio de no máximo $2 \mathrm{~km}$ de distância da UFMT.

As demais viagens são das zonas mais distantes da UFMT, dentro de Cuiabá e Várzea Grande $(7,1 \%)$ ou de outros municípios $(0,4 \%)$. O resumo desta classificação está ilustrado na Figura 4.

Figura 4 - Zonas de distâncias de deslocamento em frequência relativa ao total de viagens

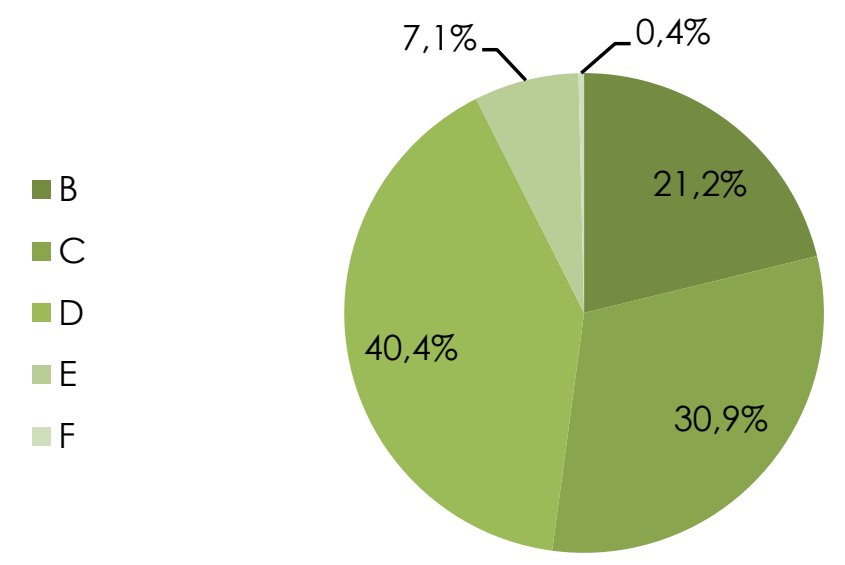

Fonte: Autor (2019)

Quanto à classificação do deslocamento em relação a sua natureza, pode-se afirmar que a maioria são viagens simples (78,5\%), ou seja, o usuário tem como origem e destino a residência. As outras viagens são encadeadas, isto é, são mais complexas e têm a UFMT em um par de deslocamentos de sua viagem, como mostra na Figura 5. 
Figura 5 - Natureza do deslocamento em frequência relativa ao total de viagens

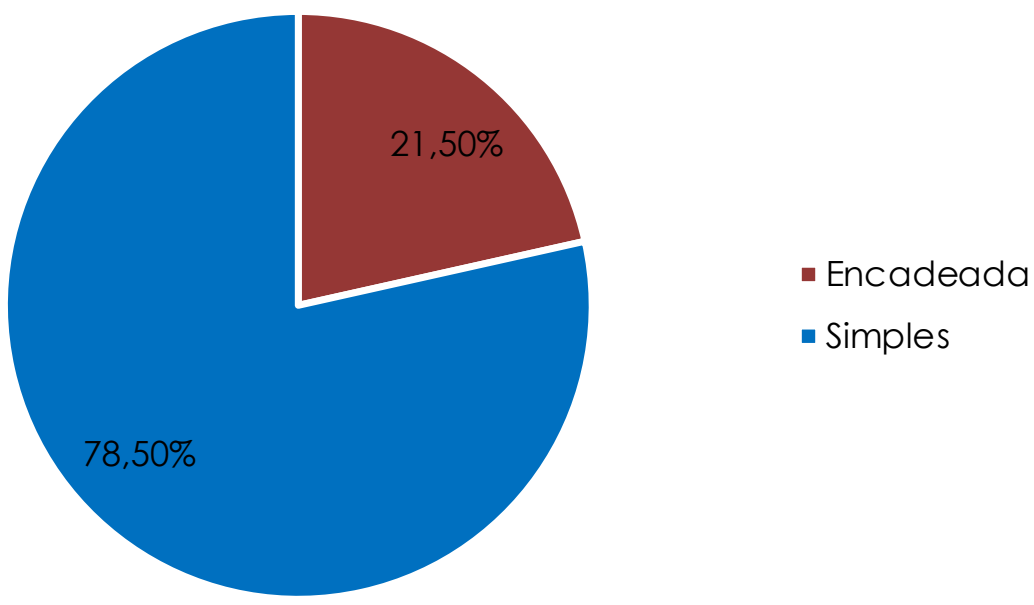

Fonte: Autor (2019)

É importante salientar que os dados, quando analisados separadamente, podem fornecer algum tipo de informação em relação aos padrões de viagens, mas é na combinação dessas três informações que é possível visualizar esses padrões de forma mais completa. A natureza das viagens dos usuários da UFMT pode ser observada em relação à distância, ao modo de transporte e a natureza da viagem, fornecendo uma visão panorâmica da mobilidade dessas pessoas com foco na frequência relativa ao total de viagens como na Figura 6, ou focada na natureza das viagens, como demonstrado na Figura 7.

A partir dessas informações é possível observar, por exemplo, que a zona B, de bairros próximos a UFMT, tem um significativo número de viagens de carro e de natureza simples. Esses usuários deveriam ser incentivados a utilizar uma modalidade de transporte ativa ou poderia ser proposto o aprofundamento da investigação dessas viagens para entender o motivo das viagens serem realizadas por veículo particular.

A zona $C$ tem a maioria das viagens sendo realizadas de carro e ônibus, mas, como se trata de uma distância máxima de $5 \mathrm{~km}$, elas têm potencial para se transformarem em viagens mais sustentáveis se convertidas em viagens de bicicletas.

Pode-se propor um índice de inadequação aos usuários do TC a partir da porcentagem dessas viagens em um raio de até $5 \mathrm{~km}$ em relação ao total de viagens. Somando os usuários que realizam viagens simples no modo de transporte ônibus nas zonas B e C, observa-se que $12 \%$ utilizam o TC, porém poderiam migrar para a modalidade ativa de transporte. A mesma proposição de inadequação pode ser sugerida para análise de usuários do $\mathrm{Tl}$, que se convertidas em viagens sustentáveis teriam grande influência na modalidade ativa de transporte, por representarem $21,51 \%$ das viagens.

Outras propostas de análises podem ser tratadas em relação às viagens de média e longa distâncias, como viagens simples ou encadeadas e para qualquer modo de transporte. 
Figura 6 - Zona de viagem por modo de transporte e natureza da viagem, com foco na frequência relativa ao total de viagens dado em porcentagem

- Simples Encadeada

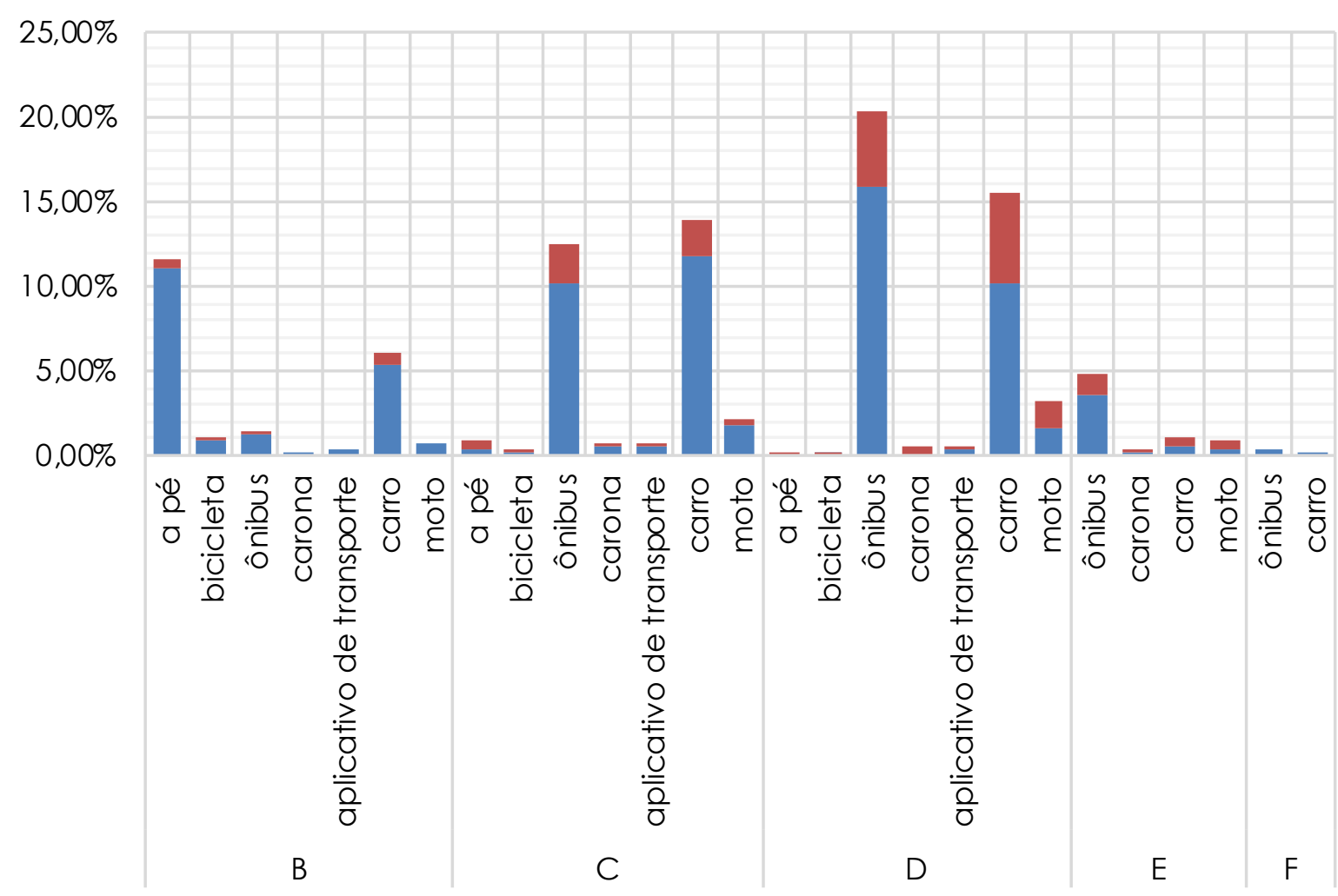

Fonte: Autor (2019) 
Figura 7 - Zona de viagem por modo de transporte e natureza da viagem, com foco na frequência de viagens relativa ao modo de transporte e zona de deslocamento, dado em porcentagem

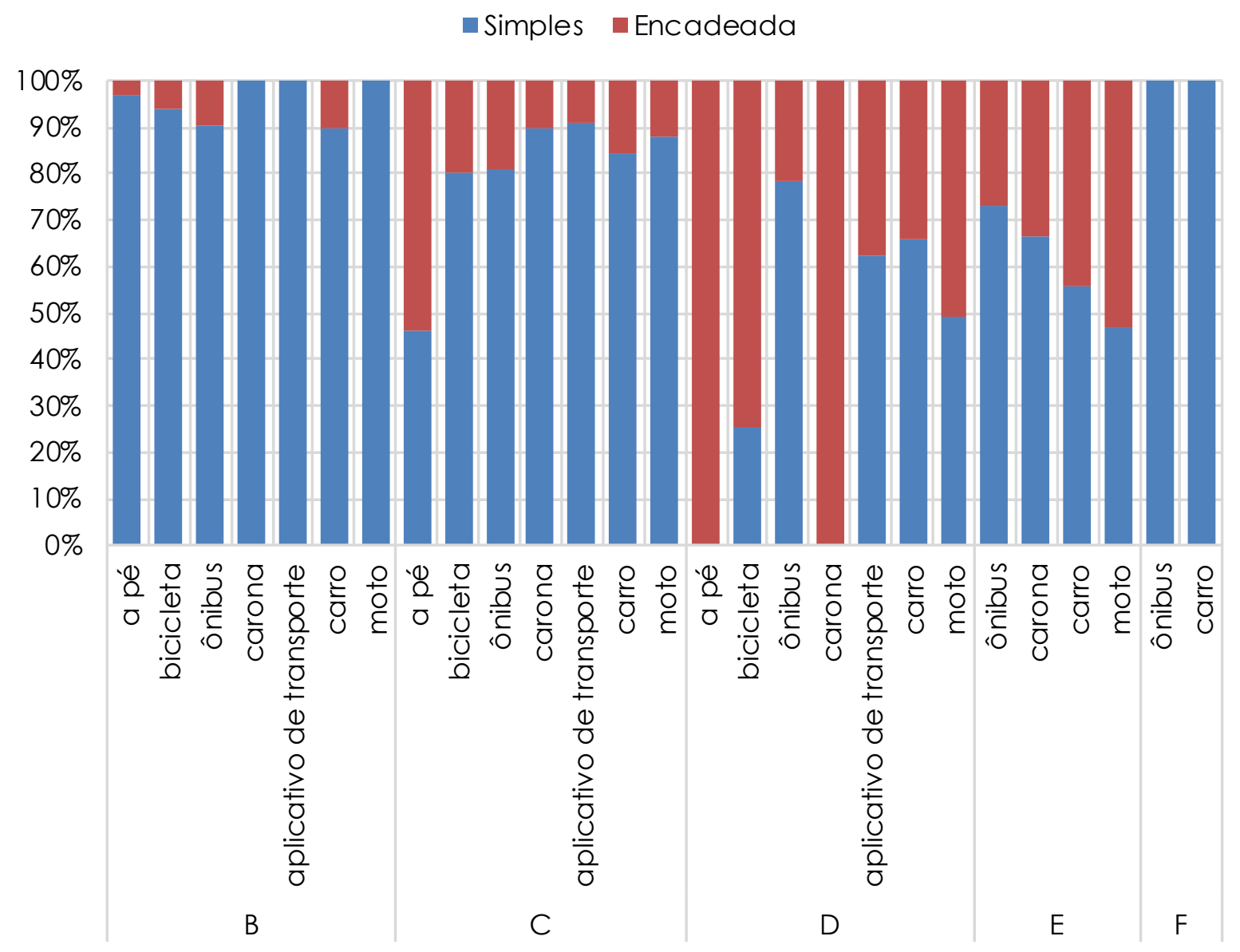

Fonte: Autor (2019)

\section{CONCLUSÃO}

O método proposto se demonstrou eficiente para analisar os padrões de viagens por levar em consideração três variáveis distintas: distância, modo e natureza do deslocamento. Estas duas últimas distribuídas de acordo com a distância.

Também é possível inferir das viagens os aspectos ligados à adequação do modo de transporte quanto aos preceitos de mobilidade sustentável. As informações obtidas por esse método de análise dão subsídios para investigação direcionada e mais personalizada a respeito dos padrões de viagens de usuários de um PGV.

Para os próximos trabalhos pretende-se aprofundar na análise dos padrões de viagens e buscar índices, ou níveis de serviço, que possam estar relacionados com as variáveis do método aplicado.

\section{REFERÊNCIAS}

ALVES, R.; BERNARDO, M.; LIMA, R. S.; LIMA, J. P. Instituições de ensino superior como polos geradores de viagem: as diferenças espaciais e temporais nos padrões de viagens. In: Anais do XXIX ANPET - Congresso de pesquisa e Ensino em Transportes, p. 2632-2643, 2015, Ouro Preto- MG. 
OLIVEIRA, A. M. Um índice para o planejamento da mobilidade com foco em grandes Polos Geradores de Viagens- Desenvolvimento e aplicação em um campus universitário. 2015. 197 f. Dissertação (Mestrado). Engenharia de Transportes, Programa de Pós-Graduação em Engenharia de Transportes, Universidade de São Paulo, São Carlos, 2015.

PASCHOAL, F. Q. G. Identificação de fatores que influenciam na escolha do modo de transporte por estudantes da Universidade de Brasília. 201855 f. Trabalho de Conclusão de Curso (Bacharelado em Engenharia Civil). Departamento de Engenharia Civil, Universidade de Brasilia, Brasilia, 2018.

PORTUGAL, L S; GOLDNER, L G. Estudo de Polos Geradores de Tráfego e de seus Impactos nos Sistemas Viários e de Transportes. São Paulo: Editora Edgard Blucher, 2003.

PREFEITURA DE CUIABÁ/SMDU/DPD. Abairramento de Cuiabá. 2010. Disponível em: <http://www.cuiaba.mt.gov.br/upload/arquivo/Abairramento.pdf>. Acesso em: 12 abr. 2019.

REDPGV. O Que é um PGV. Disponível em: <http://redpgv.coppe.ufrj.br/index.php/ptBR/conceitos/o-que-e-um-pgv>. Acesso em: 20 mar. 2019.

TAKANO, M. S. M. Análise da influência da forma urbana no comportamento de viagens encadeadas com base em padrões de atividades. 2010. 233 f. Dissertação (Mestrado). Faculdade de Tecnologia, Departamento de Engenharia Civil e Ambiental, Universidade de Brasília, Brasília, 2010.

UFMT. Anuário Estatístico 2018 - Ano base 2017. Disponível em: <http://wwwl.ufmt.br/anuarioestatistico/arquivos/del fe92638319ba0be5960867481e363.pdf >. Acesso em: 25 mar. 2019. 